NASA Technical Memorandum 100849

\title{
Electrohydrodynamic Migration of Charged Droplets in an Insulating Fluid
}

\author{
(NASA-TM-100849) ELECTROHYDRODYNAMIC N N8-21424 \\ MIGRATION OF CHARGED DROPLETS IN AN \\ INSULATING FLUID NASA) $20 \mathrm{p}$ CSCL $20 \mathrm{D}$ \\ $\begin{array}{ll}\text { Unclas } \\ \text { G3/34 } & 0140261\end{array}$
}

R. Balasubramaniam and R.A. Wilkinson

Lewis Research Center

Cleveland, Ohio

Prepared for the

National Fluid Dynamic Congress

sponsored by the American Institute of Aeronautics and Astronautics

Cincinnati, Ohio, July 24-28, 1988

\section{Nush}




\section{ELECTROHYDRODYNAMIC MIGRATION OF CHARGED DROPLETS IN AN INSULATING FLUID}

R. Balasubramaniam ${ }^{1}$ and R.A. Wilkinson

National Aeronautics and Space Administration

Lewis Research Center

Cleveland, Ohio 44135

\section{SUMMARY}

The motion of charged, conducting droplets present in an insulating fluid medium is analyzed under the action of an electric field, in microgravity. Previous analyses of this problem have considered the Maxwell stresses as the only driving force. In the present study, arguments from macroscopic thermodynamics and the molecular theory of surface tension are used to show that surface tension gradients can be induced due to the variation of the electric potential on the interface. In the limit of Reynolds numbers small compared to unity, the terminal velocity of migration of the droplet is calculated under the combined action of the Maxwell stresses and the surface tension gradients. The results show that there are no surface tension gradients (i.e., no electric potential variation at the interface) in a case that is considered strictly electrostatic. With the inclusion of surface currents due to the convection of the surface charges, surface tension gradients do exist and tend to reduce the terminal velocity of the droplet. The shape of the droplet altered by the motion has also been calculated, when the deformations from the spherical shape are small.

\section{NOMENCLATURE}

$A_{j}$ area of the interface

E electric field

Eo electric field far away from the droplet

$\mathrm{Fe}_{\mathrm{e}}, \mathrm{F}_{\mathrm{h}}$ net electric and hydrodynamic force on the droplet

f deformed droplet shape

$\mathrm{J}_{1}, \mathrm{~J}_{2}$ constants defined by equations (53) and (54)

$k_{B} \quad$ Boltzmann's constant

$k_{2}$ electrical conductivity of the droplet

$N$ normal stress

P pressure

$Q_{0}, q_{0}$ dimensional total surface charge and dimensional average surface charge density of the droplet

IResident Research Associate. 
$\tilde{q}, q$ dimensional and dimensionless surface charge density

$R, r$ dimensional and dimensionless radial coordinate

$R_{a}, R_{b}$ principal radii of curvature

Re Reynolds number

$R_{0} \quad$ droplet radius

$r_{12}$ vector joining the center of mass of two molecules

S entropy

T temperature

$\sim$

$t$ unit vector tangent to droplet surface

$U, u$ dimensional and dimensionless radial velocity

U, internal energy

$v, v$ dimensional and dimensionless tangential velocity

$V_{R}$ reference velocity

$\mathscr{V}$ volume

$V_{\infty}, V_{\infty}$ dimensional and dimensionless terminal velocity of the droplet

Y axial coordinate

$\beta \quad$ tagged constant (equals 1 or 2 )

$\Gamma_{1} \Gamma_{2}$ one and two particle (molecules) surface excess quantities

Y tagged constant (equals 0 or 1 )

$\varepsilon \quad$ electrical permittivity

$n$ dimensionless droplet deformation

$\theta \quad$ tangential coordinate

$\lambda$ ratio of lengths of axes of the droplet in the flow direction to that in the normal direction

$\mu \quad$ dynamic viscosity

$\checkmark \quad$ kinematic viscosity

$\sigma \quad$ surface tension 
$\tau, \tau^{e} \quad$ hydrodynamic and Maxwell shear stress

$\Phi \quad$ azimuthal coordinate

$\tilde{\phi}, \phi \quad$ dimensional and dimensionless electric potential

$\bar{\phi} \quad$ particle (molecular) potential energy from many particle Hamiltonian

$\tilde{\psi}, \Psi \quad$ dimensional and dimensionless stream function

Subscripts:

l outer fluid

2 droplet medium

i interface

- reference values

\section{INTRODUCTION}

Electrically charged droplets present in a fluid with which they are immiscible, will move when they are subjected to an electric field. They attain a steady speed of electrohydrodynamic migration, termed the terminal velocity, in a uniform field, when the electrical force is balanced by the viscous resistance. Such migration is also called electrokinetic or electrophoretic motion in colloidal systems, where the motion is due to the interaction of the applied filed with the electric double layer present at the interface and has been extensively investigated (refs. 1 to 6 ). Electrohydrodynamic migration, like thermocapillary migration, is useful in many applications in microgravity, where, in the absence of buoyancy, the locations of droplets and bubbles need to be controlled by various mechanisms.

There have been few investigations of the electrokinetics of fluid-fluid interfaces (ref. 6). Most studies (refs. 2 to 6) consider the Maxwell stresses as the driving force for the flow. Levich (ref. 1) has analyzed the motion of mercury drops in electrolytes, representing the driving force as a surface tension gradient, arising from the electrocapillary effect. It is the contention of the authors that the Maxwell stresses and surface tension gradients due to electrocapillarity, though each occurs because of the applied field, are independent driving mechanisms as far as the flow is concerned and hence can act simultaneously. The combined effect of the two is analyzed in this study for a model problem of the migration of a charged, conducting droplet in an electrically insulating medium. Since the droplet medium is conducting, the charge resides as a surface charge at the interface. This situation is the same as the "charge monolayer" problem considered by Spertell and Saville (ref. 4).

In what follows, the thermodynamics of the system are considered to show that surface tension gradients can be generated by the variation of the electric potential at the interface, and reasoning from a molecular basis is used to explain the origin of the surface tension gradient. The electrohydrodynamic 
formulation for the migration of a spherical droplet is then presented and the potential and flow fields and the terminal velocity are calculated. The calculated fields are lastly used to determine the slightly perturbed shape of the droplet.

\section{THERMODYNAMICS AT THE INTERFACE}

Presented below is a simplified view of the thermodynamics of the interface where the occurrence of a charge double layer is excluded. Consider a system consisting of an electrically insulating fluid with an electrically conducting immiscible droplet inside, under microgravity (fig. 1). Let the system change from one equilibrium state to another, during which the system can exchange heat and electrical charge with the surroundings, but no mass enters or leaves the system. Denoting the insulating fluid, the droplet medium and the interface region by the subscripts 1,2 and $i$ respectively and assuming that each subsystem $(1,2, i)$ remains in equilibrium at the initial and final states for a reversible process with the droplet and the interface not transferring charge to the insulating fluid, the change in the internal energy of the system is

$$
\mathrm{d} \mathscr{U}_{\mathrm{s}}=\mathrm{d} \mathscr{U}_{1}+\mathrm{d} \mathscr{U}_{2}+\mathrm{d} \mathscr{U}_{i}
$$

where

$$
\begin{gathered}
d \mathscr{U}_{1}=T_{1} d S_{1}-P_{1} d \mathscr{Y}_{1} \\
d \mathscr{U}_{2}=T_{2} d S_{2}-P_{2} d \mathscr{V}_{2} \\
d \mathscr{U}_{i}=T_{i} d S_{i}+\sigma d A_{i}+\tilde{\phi}_{i} d Q_{i}
\end{gathered}
$$

$\sigma$ is the surface tension (or interfacial tension; the two words are used interchangeably), $Q_{i}$ is the surface charge brought to the interface from infinity where the potential is zero and $\tilde{\phi}_{j}$ is the electric potential at the interface. Keeping the intensive variables fixed, if the extensive variables are permitted to undergo a finite change, (ref. 7) the following expression may be written for $U_{i}$

$$
\mathscr{U}_{i}=T_{i} S_{i}+\sigma A_{i}+\tilde{\phi}_{j} Q_{i}
$$

Differentiating equation (5) and subtracting equation (4) yields the GibbsDuhem equation for the interface as

$$
A_{i} d \sigma+Q_{j} d \tilde{\phi}_{j}+S_{j} d T_{i}=0
$$

If the surface is $i$ sothermal during the process, $d T_{i}=0$ and hence

$$
d \sigma=-\frac{Q_{i}}{A_{i}} d \tilde{\phi}_{\mathfrak{j}}
$$

which is analogous to the Lippman equation for electrocapillary phenomena (ref. 8). From equation (7), we may write

$$
\nabla_{S} \sigma=-\tilde{q}_{j} \nabla_{S} \tilde{\Phi}_{i}
$$


where $\nabla_{s}$ is the surface gradient operator and $\tilde{q}_{i}$ is the surface charge density. Thus surface tension gradients can occur when the surface is charged and the potential varies on the interface.

\section{MOLECULAR BASIS OF SURFACE TENSION GRADIENT}

If one thinks of the surface of the charged droplet as a membrane with embedded charge, then whenever there is a nonuniform charge density, there will be unbalanced electric forces between the charges. This is the physical picture that persuades us that if the externally applied electric field induces a nonuniform charge, then a surface stress is induced.

Referring to a treatise on the molecular theory of surface tension, one can write a molecular level expression for $\sigma$ as (ref. 9)

$$
\sigma=-\Gamma k_{B} T+\frac{1}{2} \int \Gamma_{2}\left(\vec{\nabla}_{r_{12}} \bar{\phi}\right) \cdot \hat{t} d \vec{r}_{12},
$$

where $\Gamma$ and $\Gamma_{2}$ are the 1 and 2 particle surface excess quantities respectively; $\bar{\phi}$ is the particle potential energy from the many particle Hamiltonian; $\hat{t}$ is a unit vector tangent to the surface; and $k_{B} T$ is the Boltzmann constant times temperature. The potential energy term above effectively accumulates the surface component of the force acting on a particle by all the other particles. $\Gamma_{2}$ ensures that contributions occur only where there is an excess or depletion of particle pairs with respect to the bulk.

On our charged surface, $\bar{\phi}$ will contain contributions from the external electric field, which is a solution to the electrostatic problem, as well as the local electric field from neighboring charged particles. It is the local electric field present in the nonuniform surface charge distribution case that we contend physically mandates a surface tension gradient in addition to Maxwell stresses calculated from an external electric field.

\section{ELECTROHYORODYNAMIC FORMULATION}

Consider a conducting droplet present in an insulating fluid of infinite extent (Fig. 1). The droplet carries a total surface charge, $Q_{0}$, and is subjected to a uniform electric field $E_{0}$. We would like to calculate the terminal velocity of the droplet and the flow and potential fields in both fluids. As the droplet moves at its constant terminal velocity, it is convenient to choose a coordinate system on the droplet with the origin at its center of mass. In this coordinate system, the outer fluid approaches the droplet with the terminal velocity $V_{\infty}$, which is to be determined.

The flow is considered to be incompressible, laminar and with spatially constant physical properties (viscosity, electrical conductivity, etc). The droplet is assumed to be spherical. From the geometry and the boundary conditions, the problem is symmetric about the flow direction (the droplet polar axis). The basic equations for the flow in the two fluids are the NavierStokes equations. The treatment of electrical effects in this study is the same as in the study by Melcher and Taylor (ref. 10). As mentioned in reference 10, the dynamic currents are so small and the induced magnetic field 
is negligible and hence, the basic equations are the electrostatic form of Maxwell's equations. It is assumed that the current density is proportional to the electric field in the droplet (Ohms law), with the electrical conductivity being a constant. In both fluids, the electric displacement is taken to be proportional to the electric field, with the permittivity being a constant. The Maxwell equations then reduce to an equation for the electrostatic potential. Subscripts 1 and 2 are used to denote the outer fluid and the droplet medium respectively. The coordinate system is $R, \theta, \Phi$ with the origin at the center of mass of the droplet (fig. I). $\theta$ is measured clockwise from the point of incidence of the flow. Rationalized MKS units are used for the electrical quantities.

A reference velocity scale $V_{R}$ for the flow field may be estimated from the fact that when the droplet moves at the terminal velocity, the Coulombic force on it is balanced by the viscous forces. Hence,

$$
4 \pi R_{0}^{2} q_{0} E_{0} \sim 4 \pi R_{0}^{2}\left(\mu_{1} V_{R} / R_{0}\right) \Rightarrow V_{R} \sim q_{O} E_{0} R_{0} / \mu 1
$$

where $q_{0}=Q_{0} /\left(4 \pi R_{0}{ }^{2}\right)$ is the surface charge density. $V_{R}$ thus yields typical values of the velocities thay may be anticipated. The Reynolds number is

$$
R e=V_{R} R_{0} / v=q_{O} E_{0} R_{0}^{2} /(\mu, v)
$$

Re compares the inertial forces on a fluid element to the viscous forces on it. In what follows, Re for both fluids is assumed to be small compared to unity. The Navier-Stokes equations are written in the Re $<1$ limit for the stream function (ref. 11). Dimensionless variables are defined as follows

$$
r=\frac{R}{R_{0}}, u=\frac{U}{V_{R}}, v=\frac{V}{V_{R}}, \psi=\frac{\tilde{\psi}}{V_{R} R_{0}^{2}}, \phi=\frac{\tilde{\phi}}{E_{0} R_{0}}
$$

where $V_{R}$ is as defined by equation (10) and the stream function $\psi$ is such that

$$
u=-\frac{1}{r^{2} \sin \theta} \frac{\partial \psi}{\partial \theta}, v=\frac{1}{r \sin \theta} \frac{\partial \psi}{\partial r}
$$

The basic equations in the two fluids are

$$
\begin{aligned}
& D^{4} \psi=0 \\
& \nabla^{2} \phi=0
\end{aligned}
$$

where

$$
\left.\begin{array}{c}
D^{2}=\frac{\partial^{2}}{\partial r^{2}}+\frac{1}{r^{2}} \sin \theta \frac{\partial}{\partial \theta}\left[\frac{1}{\sin \theta} \frac{\partial}{\partial \theta}\right] \\
\nabla^{2}=\frac{1}{r^{2}} \frac{\partial}{\partial r}\left[r^{2} \frac{\partial}{\partial r}\right]+\frac{1}{r^{2} \sin \theta} \frac{\partial}{\partial \theta}\left[\sin \theta \frac{\partial}{\partial \theta}\right]
\end{array}\right]
$$


The boundary conditions are $r \rightarrow \infty$ are

$$
\psi_{1} \rightarrow \frac{v_{\infty}}{2} r^{2} \sin ^{2} \theta
$$

i.e., the velocity tends to the free stream velocity $v_{\infty}=v_{\infty} / V_{R}$ and

$$
\phi_{1} \rightarrow-r \cos \theta
$$

i.e., the electric field is uniform far away from the $\operatorname{droplet}\left(\vec{E}=-\nabla \tilde{\phi}=-E_{O} \widehat{Y}\right)$.

The boundary conditions at $r=1$ (the droplet surface) are

$$
\psi_{1}=\text { constant }=0, \psi_{2}=\text { constant }=0
$$

i.e., the radial velocities are zero at the surface. The constants may be set equal to zero without loss of generality.

$$
\frac{\partial \psi_{1}}{\partial r}=\frac{\partial \psi_{2}}{\partial r}
$$

i.e., the tangential velocities are continuous,

$$
\frac{\partial \phi_{1}}{\partial \theta}=\frac{\partial \phi_{2}}{\partial \theta}
$$

i.e., the tangential fields are continuous,

(d)

$$
\tilde{q}=\left.\left[-\varepsilon_{1} \frac{\partial \tilde{\phi}_{1}}{\partial R}+\varepsilon_{2} \frac{\partial \tilde{\phi}_{2}}{\partial R}\right]\right|_{R=R_{0}}
$$

This boundary condition is written dimensionally and represents the discontinuity in the normal displacement fields in proportion to the surface charge density (ref. 12). Since the total charge on the droplet is $Q_{0}=4 \pi R_{0}^{2} q_{0}$, we must have

$$
\int_{0}^{\pi} \tilde{q} 2 \pi R_{0}^{2} \sin \theta d \theta=4 \pi R_{0}^{2} q_{0}
$$

(e) Shear stress balance: The balance of shear stresses at the interface may be written as

$$
\tau_{1 R \theta}-\tau_{2 R \theta}+\tau_{1 R \theta}^{e}-\tau_{2 R \theta}^{e}+\frac{1}{R_{0}} \frac{\partial \sigma}{\partial \theta}=0
$$

where $\tau_{R \theta}=\left.\mu R \frac{\partial}{\partial R}\left[\frac{V}{R}\right]\right|_{R=R_{0}}$ is the hydrodynamic shear stress and $\tau_{R \theta}^{e}=\left.\varepsilon E_{R} E_{\theta}\right|_{R=R_{0}}$ is the electric or Maxwell shear stress. From equation (8), 
$\partial \sigma / \partial \theta=-\tilde{q} \partial \tilde{\phi} / \partial \theta$. Using dimensionless variables and defining $q=\tilde{q} / q_{0}$, the shear stress balance at $r=1$ may be written as

$$
\frac{\partial}{\partial r}\left[\frac{v_{1}}{r}\right]-\frac{\mu_{2}}{\mu_{1}} \frac{\partial}{\partial r}\left[\frac{v_{2}}{r}\right]=2 q \frac{\partial \phi}{\partial \theta}
$$

It may be noted that $\tau_{1} \stackrel{e}{r} \theta-\tau_{2} \underset{r}{e} \theta=\partial \sigma / \partial \theta=-q(\partial \phi / \partial \theta)$ at $r=1$ and hence the coefficient in the right hand side of equation (24) is 2 . Since this is the only place in the formulation that the surface tension gradients are encountered (surface tension is also encountered in the normal stress balance at the droplet surface and will be addressed later), the term $2 q \partial \phi / \partial \theta$ will be tagged for purposes of comparison and written as $\beta q \partial \phi / \partial \theta$. Hence $\beta=2$ will recover the present formulation while $\beta=1$ represents a formulation where either Maxwell shear stresses or surface tension gradients (but not both) are considered.

(f) Charge conservation: Since the interface can move with respect to the bulk of the droplet, the motion of the surface charges constitutes an electric current at the surface. Since charge must be conserved, and the outside medium is insulating, there must exist a radial conduction current at the surface from the inside medium. Using Ohm's Iaw (current density = conductivity $x$ field) and inventorying charge movement, charge conservation may be written as

$$
\frac{\partial \phi_{2}}{\partial \theta}=-\frac{q_{0}^{2}}{k_{2} \mu_{1}} \frac{1}{\sin \theta} \frac{\partial}{\partial \theta}(q v \sin \theta)
$$

(g) Net force on the droplet: As the droplet moves at its terminal velocity, the net force acting on it must be zero. Since surface tension is an internal force for the droplet and cannot act on itself, the sum of the hydrodynamic and electrical forces exerted on the droplet by the outside medium must be zero, i.e., $\vec{F}_{e}+\vec{F}_{h}=0$. By symmetry, the net force perpendicular to the flow direction is zero. That the net force parallel to the flow direction is zero may be expressed as

$$
\begin{aligned}
\int_{0}^{\pi}\left\{\left[\varepsilon_{1} E_{R 1} E_{\theta 1} \sin \theta-\right.\right. & \left.\frac{\varepsilon_{1}}{2} E_{R 1}^{2} \cos \theta\right]+\left[\mu_{1} R_{0} \frac{\partial}{\partial R}\right. \\
& \left.\left.\times\left(\frac{V_{1}}{R}\right) \sin \theta-\left(2 \mu, \frac{\partial U_{1}}{\partial R}-P_{1}\right) \cos \theta\right]\right\}_{R=R_{0}} \sin \theta d \theta=0
\end{aligned}
$$

where $P_{1}$ is the hydrodynamic pressure in the outer fluid. This condition is essentialiy what determines the unknown eigenvalue $V_{\infty}$. Instead of integrating the surface stresses, $F_{e}$ may also be calculated alternatively by recognizing that it is equal to the force on the net charge $Q_{0}$ due to the uniform field $E_{O}$. Hence

$$
F_{e}=-4 \pi R_{o}^{2} q_{0} E_{0}
$$


in the $\hat{Y}$ direction, where $Y=-R \cos \theta$. Similarly, $F_{h}$ may also be calculated by using a theorem (ref. 11) valid when $\mathrm{Re}<<1$ and may be expressed in dimensional form along $\hat{\gamma}$ direction as

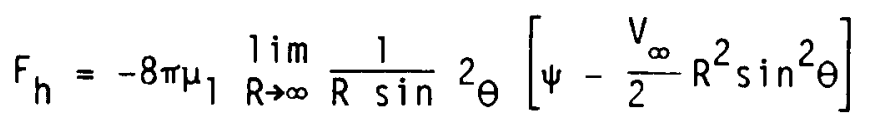

This completes the specification of the boundary value problem. It may be noted that the normal stress balance equation at the droplet surface has not been used in the formulation thus far. This is because the droplet is assumed to be spherical. Strictly speaking, it is the normal stress balance that determines the true shape of the droplet as it moves. We will return to this point later.

\section{SOLUTION}

(a) Case 1: Consider a case where $q_{0}^{2 / k_{2} \mu_{1}}<1$ as is the case for highly conducting droplets. The surface current due to charge convection may then be neglected and the problem is strictly electrostatic. Equation (25) reduces to

$$
\frac{\partial \phi_{2}}{\partial r}=0 \quad \text { at } \quad r=1
$$

The solution to equations (13) and (14) is

$$
\begin{gathered}
\phi_{1}=-r \cos \theta+\frac{q_{0}}{\varepsilon_{1} E_{0}} \frac{1}{r}+\frac{\cos \theta}{r^{2}} \\
\phi_{2}=c_{1}=\text { constant } \\
\psi_{1}=\left[-\frac{r}{2}+\frac{v_{\infty}}{2} r^{2}+\frac{1}{2}\left(1-v_{\infty}\right) \frac{1}{r}\right] \sin ^{2} \theta \\
\Psi_{2}=\frac{1}{4}\left(3 v_{\infty}-2\right)\left(r^{4}-r^{2}\right) \sin ^{2} \theta \\
v_{\infty}=\left[1+\frac{\mu_{2}}{\mu_{1}}\right] /\left[1+\frac{3 \mu_{2}}{2 \mu_{1}}\right]
\end{gathered}
$$

The dimensional terminal velocity is

$$
V_{\infty}=\frac{q_{0} E_{0} R_{0}\left[1+\frac{\mu_{2}}{\mu_{1}}\right]}{\mu_{1}\left[1+\frac{3 \mu_{2}}{2 \mu_{1}}\right]}
$$

which is the same as in reference 4 .

When $\mu_{2} \rightarrow \infty$, there is no flow in the droplet and the results reduce to the motion of a solid sphere in a fluid. The terminal velocity of the solid 
sphere may be derived by equating the Coulombic force on it to the Stokes drag. This yields $4 \pi R_{O}^{2} q_{O} E_{O}=6 \pi R_{O} \mu 1 V_{\infty}, V_{\infty}=2 q_{O} E_{O} R_{O} /\left(3 \mu_{1}\right)$, which checks with equation (35) in the limit $\mu_{2} \rightarrow \infty$. From the solution, since $\phi_{2}=C_{1}$ and $\partial \phi_{1} / \partial \theta=0$ at $r=1$, there are no tangential Maxwell stresses and no surface tension gradients at the surface of the droplet. All the driving force for the flow comes from the normal Maxwell stresses, which is what contributes to $\mathrm{Fe}_{\mathrm{e}}$. Since the normal stress condition at $r=1$ has not been considered, the driving force is not explicitly seen. To see the effects of surface tension gradients, which is the primary goal of the present study, another case must be considered where charge convection must be retained.

(b) Case 2: Here $q_{0}{ }^{2 / k_{2} \mu l}$ is not small and charge convection is retained. The resulting nonuniform charge distribution will induce surface tension gradients ( $c f$, the molecular picture). However, for mathematical simplicity, the surface charge distribution is taken to be uniform in the charge conservation boundary condition, i.e., the change in the surface charge density is much smaller than the average surface charge density $q_{0}$ and $\partial q / \partial \theta$ is negligible. The exact condition, when this is true, is given following equation (40). For this case $q \approx 1$ and equation (25) becomes

$$
\frac{\partial \phi_{2}}{\partial r}=-\frac{q_{0}^{2}}{k_{2} \mu_{1}} \frac{1}{\sin \theta} \frac{\partial}{\partial \theta}(v \sin \theta) \text { at } r=1
$$

The solution is

$$
\begin{gathered}
\phi_{1}=-r \cos \theta+\frac{q_{0}}{\varepsilon_{1} E_{0}} \frac{1}{r}+\left[1-\frac{q_{0}^{2}}{k_{2} \mu_{1}}\left(3 v_{\infty}-2\right)\right] \frac{\cos \theta}{r^{2}} \\
\phi_{2}=-\frac{q_{0}^{2}}{k_{2} \mu_{1}}\left(3 v_{\infty}-2\right) r \cos \theta \\
v_{\infty}=\frac{\left[3+\frac{2 \beta q_{0}^{2}}{k_{2} \mu_{1}}+\frac{3 \mu_{2}}{\mu_{1}}\right]}{\left[3+\frac{3 \beta q_{0}^{2}}{k_{2} \mu_{1}}+\frac{9 \mu_{2}}{2 \mu_{1}}\right]}
\end{gathered}
$$

$\psi_{1}$ and $\psi_{2}$ are the same as in equations (32) and (33), with $v_{\infty}$ as given above. The surface charge density calculated from equations (21) and (37) to (39) is

$$
q=1+\frac{\varepsilon_{1} E_{0}}{q_{0}}\left[3-\frac{\frac{q_{0}^{2}}{k_{2} \mu_{1}}\left[2+\frac{\varepsilon_{2}}{\varepsilon_{1}}\right]}{1+\frac{\beta q_{0}}{k_{2} \mu_{1}}+\frac{3 \mu_{2}}{2 \mu_{1}}}\right] \cos \theta
$$


Thus $q \approx 1$ is typically the case when $\varepsilon_{1} E_{O}, \varepsilon_{2} E_{0} \ll q_{0}$. It may be noted that in the limit $q_{0} 2 /\left(k_{2} \mu\right) \rightarrow 0$, equation (39) becomes equation (34) and the solution for Case 1 is recovered. Also $v_{\infty} \rightarrow 2 / 3$ when $\mu_{2} / \mu_{1} \rightarrow \infty$, which is the result for a solid sphere. It is interesting to note that $v_{\infty}$ also becomes

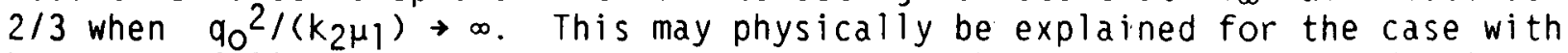
$k_{2} \rightarrow 0$ as follows. In equations (25) or (36), when $k_{2} \rightarrow 0$, the conduction current at the droplet surface tends to zero, as we expect finite electric fields within the droplet. Hence, the divergence of the current due to charge convection, the charge convection current iteself, and hence the surface velocity must be zero. This is precisely the case for a nonconducting solid sphere, where the surface velocity is zero on account of the no slip condition. In Case 2, driving forces due to normal and tangential Maxwell stresses at the surface and surface tension gradients are all present. As mentioned before, the effects of the surface tension gradients are contained in the tagged variable $\beta . \quad \beta=2$ includes surface tension gradients, while $\beta=1$ does not $(\beta=1$ may also represent a case where surface tension gradients are retained while Maxwell stresses are not; however, the Maxwell stresses must then be neglected everywhere in the formulation and would result in $F_{e} \equiv 0$ in the consideration of the net force on the droplet, a result that is not physically sensible). From equation (39),

$$
\frac{\partial v_{\infty}}{\partial \beta}=-\frac{q_{0}^{2}}{k_{2} \mu_{1}} /\left[1+\frac{\beta q_{0}^{2}}{k_{2} \mu_{1}}+\frac{3 \mu_{2}}{2 \mu_{1}}\right]^{2}
$$

Thus, for any $\beta, \partial v_{\infty} / \partial \beta$ is always negative. Hence $v_{\infty}$ is smaller for $\beta=2$ than for $\beta=1$, and so all other things held constant, inclusion of surface tension gradients results in a lower terminal velocity of migration of the droplet. The dimensional terminal velocity including surface tension gradients is

$$
v_{\infty}=\frac{q_{0} E_{0} R_{0}}{\mu_{1}} \frac{\left[3+\frac{4 q_{0}{ }^{2}}{k_{2} \mu_{1}}+\frac{3 \mu_{2}}{\mu_{1}}\right]}{\left[3+\frac{6 q_{0}}{k_{2} \mu_{1}}+\frac{9 \mu_{2}}{2 \mu_{1}}\right]}
$$

\section{(c) Deformations of the droplet:}

Having determined the terminal velocity and the velocity and potential fields, the perturbations to the shape of the droplet may now be determined, assuming that such deformations are small. As mentioned before, it is the normal stress balance at the droplet surface that determines its true shape and will now be considered. The following analysis closely resembles that in references 13 and 14 . The true shape of the droplet is represented by $R(\theta)=R_{0}+f(\theta)$. The normal stress condition may be written as

$$
N_{1}-N_{2}=\sigma\left[\frac{1}{R_{a}}+\frac{1}{R_{b}}\right] \text { at } R=R_{0}+f
$$

where $N$ is the normal stress due to both hydrodynamic and electrical effects and $R_{a}, R_{b}$ are the principal radi $i$ of curvature of the interface. When $n=f / R_{0} \ll 1$, the following may be written (ref. 13) 


$$
\begin{gathered}
N=\tau_{R R}+\tau_{R R}^{e}-\frac{2}{R_{0}+f}\left(\tau_{R \theta}+\tau_{R \theta}^{e}\right) \frac{d f}{d \theta} \\
\frac{1}{R_{d}}+\frac{1}{R_{b}}=\frac{2}{R_{0}}-\frac{2 f}{R_{o}^{2}}-\frac{1}{R_{o}^{2} \sin \theta} \frac{d}{d \theta}\left[\sin \theta \frac{d f}{d \theta}\right]
\end{gathered}
$$

where $\tau_{R R}=-P+2 \mu \partial U / \partial R, \tau_{R R} e_{R R}=\varepsilon / 2\left(E_{R}^{2}-E_{\theta}^{2}\right), P$ being the hydrodynamic pressure. The dimensionless pressure $P=P /\left(\mu V_{R} / R_{0}\right)$ in the two fluids may be shown to be (ref. 1)

$$
p_{1}=-\frac{2 A}{r^{2}} \cos \theta, p_{2}=K_{0}-20 A_{1} r \cos \theta
$$

where $A$ and $A_{1}$ are the coefficients of $r \sin ^{2} \theta$ and $r^{4} \sin ^{2} \theta$ terms of the respective stream functions and $K_{0}$ is an unknown constant. The surface tension $\sigma$ is a function of $\theta$ and from equation (8), it may be written as

$$
\sigma(\theta)=\sigma_{0}-\gamma \tilde{q}\left[\tilde{\phi}\left(R_{0}, \theta\right)-\tilde{\phi}_{0}\right]
$$

where $\tilde{q}$ has been assumed to be independent of $\theta . \quad y$ is again a tagged variable, where $Y=1$ retains the surface tension variations, while $Y=0$ regards the surface tension as uniform.

Droplet shape for Case 1: Assuming $n<1$ and using equations (44) to (47) and the solutions to $\phi$ and $\psi$ for Case 1, equation (43) may finally be written as

$$
\frac{1}{\sin \theta} \frac{d}{d \theta}\left[\sin \theta \frac{d \eta}{d \theta}\right]+2 n=2-\frac{\mu_{2} V_{R}}{\sigma_{0}} k_{0}-\frac{q_{0}{ }^{2} R_{0}}{2 \varepsilon_{1} \sigma_{0}}-\frac{9}{2} \frac{\varepsilon_{1} E_{0}{ }^{2} R_{0}}{\sigma_{0}} \cos ^{2} \theta
$$

where the left hand side of equation (48) is essentially the right hand side of equation (43). Neither $\beta$ nor $\gamma$ appear in equation (48) as $\partial \phi / \partial \theta=0$ at $r=1$ for Case 1. The boundary conditions on $n$ are that the volume of the droplet is unchanged as it deforms and the origin of the coordinates is at the center of mass of the droplet. For $n \ll 1$, these become

$$
\int_{0}^{\pi} n \sin \theta d \theta=0, \quad \int_{0}^{\pi} n \cos \theta \sin \theta d \theta=0
$$

From the analysis by Brignell (ref. 15), the solution is

$$
n(\theta)=\frac{3}{8} \frac{\varepsilon_{1} E_{0}^{2} R_{0}}{\sigma_{0}}\left(3 \cos ^{2} \theta-1\right)
$$

Hence, $n \ll 1$ is valid when $\varepsilon_{1} E_{0}{ }^{2} R_{0} / \sigma_{0} \ll<1$. The shape of the droplet is an ellipsoid of revolution, with an axis of rotation along the flow direction. To leading order, the ratio of the lengths of axes in the flow direction to that in the normal direction is 


$$
\lambda=\left[1+\eta\left(0^{\circ}\right)\right] /\left[1+\eta\left(90^{\circ}\right)\right] \approx 1+\frac{9 \varepsilon_{1} E_{O}{ }^{2} R_{0}}{8 \sigma_{0}}
$$

One sees, therefore, the droplet elongates in the flow direction.

Droplet shape for Case 2: Similarly, using the solutions for Case 2 and assuming that $q_{0} E_{0} R_{0} / \sigma_{0} \ll 1$ (i.e., $\left.\Delta \sigma / \sigma_{0} \ll 1\right)$ and $n \ll 1$, equation (43) may finally be written for the combinations $\beta=2, \gamma=1$ or $\beta=1, \gamma=0$ as

$$
\frac{1}{\sin \theta} \frac{d}{d \theta}\left[\sin \theta \frac{d \eta}{d \theta}\right]+2 \eta=2-\frac{\mu_{2} V_{R}}{\sigma_{0}} K_{0}-\frac{\varepsilon_{1} E_{0}^{2} R_{0}}{2 \sigma_{0}}\left[J_{2}+\left(J_{1}-J_{2}\right) \cos ^{2} \theta\right]
$$

where

$$
\begin{gathered}
J_{1}=9+12 \frac{q_{0}^{2}}{k_{2} \mu_{1}}\left(2-3 v_{\infty}\right)+\left[4-\frac{\varepsilon_{2}}{\varepsilon_{1}}\right] \frac{q_{0}^{4}}{k_{2}^{2} \mu_{1}^{2}}\left(2-3 v_{\infty}\right)^{2} \\
J_{2}=\left[\frac{\varepsilon_{2}}{\varepsilon_{1}}-1\right] \frac{q_{0}^{4}}{k_{2}^{2} \mu_{1}^{2}}\left(2-3 v_{\infty}\right)^{2}
\end{gathered}
$$

It is interesting to note that for both the combinations of $\beta$ and $\gamma$, no cos $\theta$ terms are present in the right hand side of equation (52). Y does not appear anywhere else in equations (52) to (54) when $\Delta_{\sigma} / \sigma_{0} \ll 1$. The presence of a $\cos \theta$ term causes a logarithmic singularity in the solution for $n$ at $\theta=0, \pi$. Hence, both the formulations with and without surface tension gradi ents predict finite deformations of the droplet. The solution is

$$
\eta(\theta)=\frac{1}{24} \frac{\varepsilon_{1} E_{0}^{2} R_{0}}{\sigma_{0}}\left(J_{1}-J_{2}\right)\left(3 \cos ^{2} \theta-1\right)
$$

Once again, $n \ll l \Rightarrow \varepsilon_{1} E_{0}{ }^{2} R_{0} / \sigma_{0} \ll 1$. However, $q_{0} E_{O} R_{0} / \sigma_{0} \ll 1$ that has been assumed is a more restrictive condition as $\varepsilon_{j} E_{O} / q_{0}<1$ for case 2 . It may

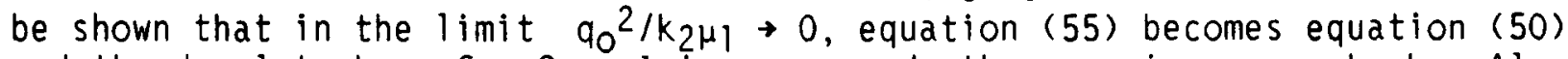
and the droplet shape for Case 1 is recovered, thus serving as a check. Also.,

$$
\frac{\partial \eta}{\partial \beta}=\frac{\varepsilon_{1} E_{0}^{2} R_{0}}{24 \sigma_{0}}\left(3 \cos ^{2} \theta-1\right) \frac{\frac{12 q_{0}{ }^{4}}{\left(k_{2} \mu_{1}\right)^{2}}\left[1+\frac{3 \mu_{2}}{2 \mu_{1}}\right]+\frac{q_{0}}{\left(k_{2} \mu_{1}\right)^{3}}\left[12 \beta-10+\frac{4 \varepsilon_{2}}{\varepsilon_{1}}\right]}{\left[1+\frac{\beta q_{0}}{k_{2} \mu_{1}}+\frac{3 \mu_{2}}{2 \mu_{1}}\right]^{3}}
$$


and $\partial|n| / \partial \beta>0$ for $\beta>5 / 6-\varepsilon_{2} /\left(3 \varepsilon_{1}\right)$. Hence, $|\eta|$ for $\beta=2$ is greater than $|n|$ for $\beta=1$. Thus the inclusion of surface tension gradients increases the deformations of the droplet. Physically, this is to be expected as surface tension variations would tend to deform the droplet further away from its spherical shape. Under the conditions $\Delta \sigma / \sigma_{0} \ll 1$ and $n<1$, the increase in deformations comes from a greater imbalance in the radial Maxwell stresses at the surface of the droplet, originating from the inclusion of surface tension variations. To leading order, the ratio of lengths of axes of the droplet in the flow direction to that in the normal direction is

$$
\lambda \approx 1+\frac{\varepsilon_{1} E_{0}{ }^{2} R_{0}}{8 \sigma_{0}}\left(J_{1}-J_{2}\right)
$$

\section{DISCUSSION}

It has been assumed that the charge exists as a surface charge in the droplet. This is strictly true only when there is no flow and may be assumed to be approximately true when the convection of charge is small compared to charge diffusion in the bulk of the droplet. That is, charge conduction restores surface charge much faster than surface convection disrupts it. From the charge continuity equation in the bulk (ref. 10), this may be shown to be valid when $\varepsilon_{2} V_{R} /\left(k_{2} R_{0}\right)$, i.e., $q_{0} E_{0} \varepsilon_{2} /\left(k_{2} \mu_{1}\right)$, i.e., $q_{0}{ }^{2} /\left(k_{2} \mu_{1}\right) \times \varepsilon_{2} E_{O} / q_{0} \ll 1$. Since $\varepsilon_{2} E_{0} / q_{0}$ must be small to justify the assumption of uniform surface charge distribution (eq. (40)), this imposes a limit on how big $q_{0} 2 /\left(k_{2} \mu_{j}\right)$ can be.

Figure 2 shows the terminal velocity $v_{\infty}$ (eq. (39)) for various values of $\mu_{2} / \mu_{1}$ and $q_{0} 2 /\left(k_{2} \mu_{1}\right)$ with $\beta=2$. As can be seen, $v_{\infty}$ is of order one for all cases thus indicating that the reference scale chosen in appropriate. Also, $v_{\infty}$ tends to $2 / 3$, the value for a solid sphere, for large values of

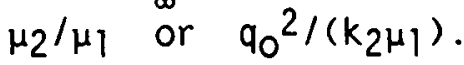

Figure 3 shows the shape of the droplet $(e q .(55))$ for $\mu_{2} / \mu_{1}=0.1$, $\varepsilon_{1} E_{O}^{2} R_{0} / \sigma_{O}=0.1$ and various values of $q_{0} 2 /\left(k_{2} \mu_{1}\right)$ and $\varepsilon_{2} / \varepsilon_{1}$. When $q_{o}{ }^{2}\left(k_{2 \mu l}\right)=0$, the shape is independent of $\varepsilon_{2} / \varepsilon_{l}$, which is consistent with equation $(50)$, the result for Case 1. The droplet can be elongated or contracted in the flow direction, the latter being the case for large values of $\varepsilon_{2} / \varepsilon_{1}$ and $q_{0} 2 /\left(k_{2} \mu_{1}\right)$. The perturbations from a spherical shape must however be small for the theory to be valid. Many of the assumptions made are true for small values of $E_{0}$; hence, this theory is essentially a small field strength theory.

\section{SUMMARY}

The aim of this study is to examine the effects of surface tension variations due to electrical effects on the migration of droplets in an insulating medium under microgravity condition. The important results are that surface tension gradients exist only when charge convection is included and tends to decrease the migration velocity and increase the extent of deformations of the droplet. The assumptions that the Reynolds number of the motion is small compared to one and that the charge redistribution is negligible essentially restricts the theory to small applied field strengths. Further analysis must 
be performed to include the effects of electric double layers at the droplet surface, non-negligible Reynolds numbers and the redistribution of charge on the migration velocity and shape of the droplet.

\section{REFERENCES}

1. Levich, V.G., Physicochemical Hydrodynamics, Prentice Hall, Englewood Cliffs, NJ, 1962 .

2. Levine, S. and O'Brien, R.N., "A Theory of Electrophoresis of Charged Mercury Drops in Aqueous Electrolyte Solution," Journal of Colloid and Interface Science, Vol. 43, No. 3, June 1973, pp. 616-629.

3. O'Brien, R.W. and White, L.R., "Electrophoretic Mobility of a Spherical Colloidal Particle," Journal of the Chemical Society, Faraday Transactions II, Vol. 74, No. 9, 1978, pp. 1607-1626.

4. Spertell, R.B. and Saville, D.A., "The Roles of Electrohydrodynamic Phenomena in the Motion of Drops and Bubbles, "Proceedings of the International Colloquium on Drops and Bubbles, Vol. I, D.J. Collins, M.S. Plesset, and M.M. Saffren, eds., Jet Propulsion Lab, California Institute of Technology, Pasadena, CA, 1974, pp. 106-121.

5. Saville, D.A., "Electrokinetic Effects with Small Particles," Annual Review of Fluid Mechanics, Vol. 9, M. Van Dyke and J.V. Wehausen, eds., Annual Reviews Inc., Palo Alto, CA, 1977, pp. 321-337.

6. Hunter, R.J., Zeta Potential in Colloid Science-Principles and Applications, Academic Press, New York, 1981.

7. Callen, H.B., Thermodynamics, John Wiley and Sons Inc., New York, 1960.

8. Hiemenz, P.C., Principles of Colloid and Surface Chemistry, Marcel Dekker Inc., New York, 1977.

9. Ono, S. and Kondo, S., "Molecular Theory of Surface Tension in Liquids," Handbuck der Physik, Vol. 10, S. Flugge, ed., Springer Verlag, Berlin, 1960, pp. 134-280.

10. Melcher, J.R. and Taylor, G.I., "Electrohydrodyanmics: A Revlew of the Role of Interfacial Shear Stresses," Annual Review of Fluid Mechanics, Vol. 1, W.R. Sears and M. Van Dyke, eds., Annual Reviews Inc., Palo Alto, CA, 1969, pp. 111-146.

11. Happe 1, J. and Brenner, H., Low Reynolds Number Hydrodynamics, Noordhoff Int1. Publishing, Leyden, Netherlands, 1973.

12. Jackson, J.D., Classical Electrodynamics, John Wiley \& Sons Inc., New York, 1962.

13. Taylor, T.D. and Acrivos, A., "On the Deformation and Drag of a Falling Viscous Drop at Low Reynolds Number," Journal of Fluid Mechanics, Vol. 18 , Mar. 1964, pp. 466-476. 
14. Balasubramanlam, R. and Chai, A.T., "Thermocapillary Migration of Droplets: An Exact Solution for Small Marangoni Numbers, "Journal of Colloid and Interface Science, Vol. 119, Oct. 1987, pp. 531-538.

15. Brigne11, A.S., "The Deformation of a Liquid Drop at Small Reynolds Number," Quarterly Journal of Mechanics and Applied Mathematics, Vol. 26, Pt. 1, Feb, 1973, pp. 99-107.

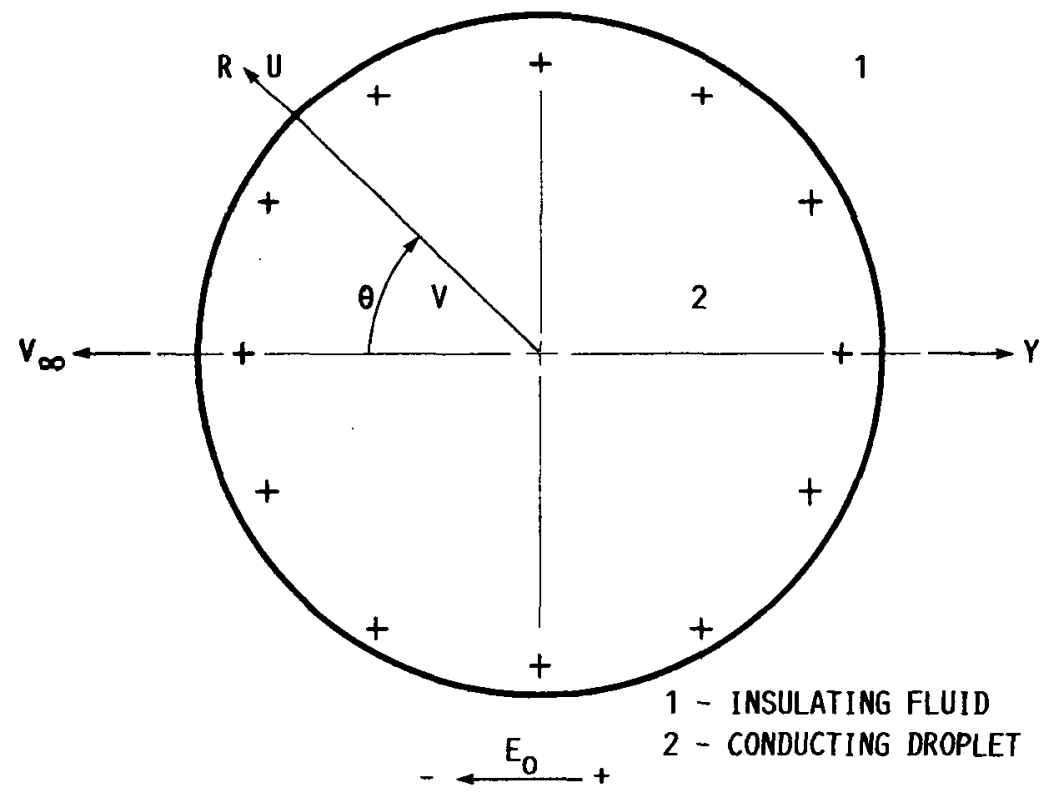

FIGURE 1. - SKETCH OF A MIGRATING DROPLET. 


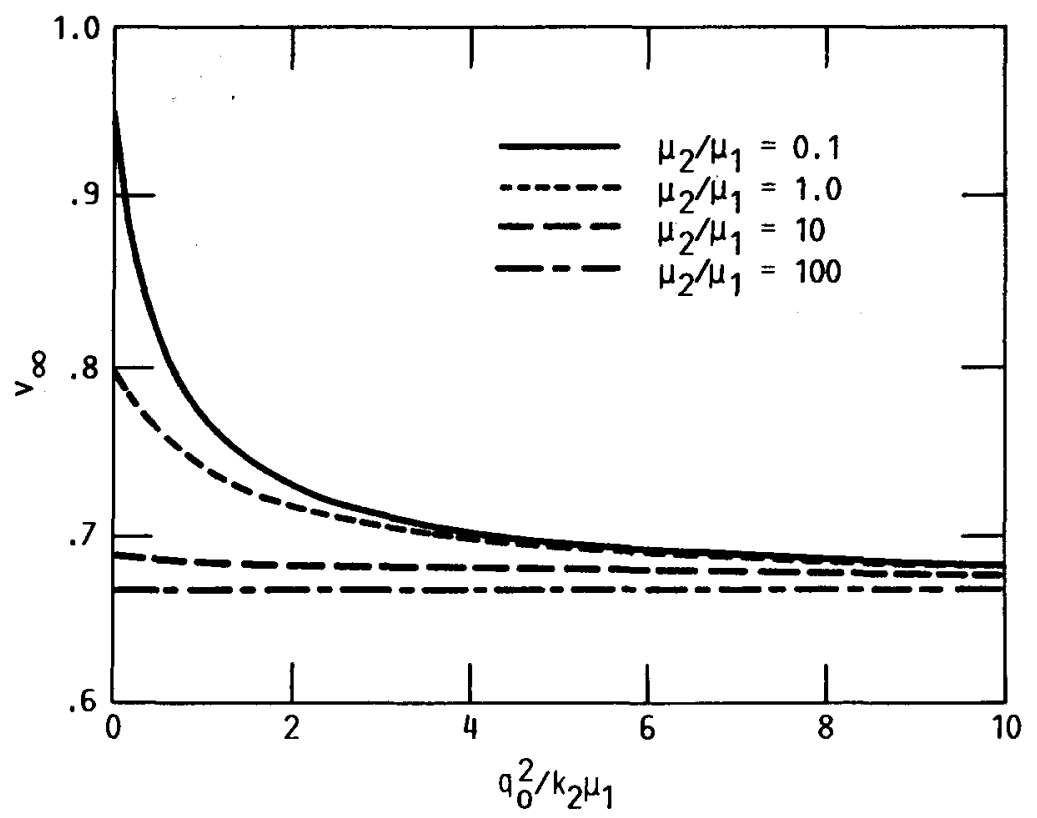

FIGURE 2. - DROPLET TERMINAL VELOCITIES. 


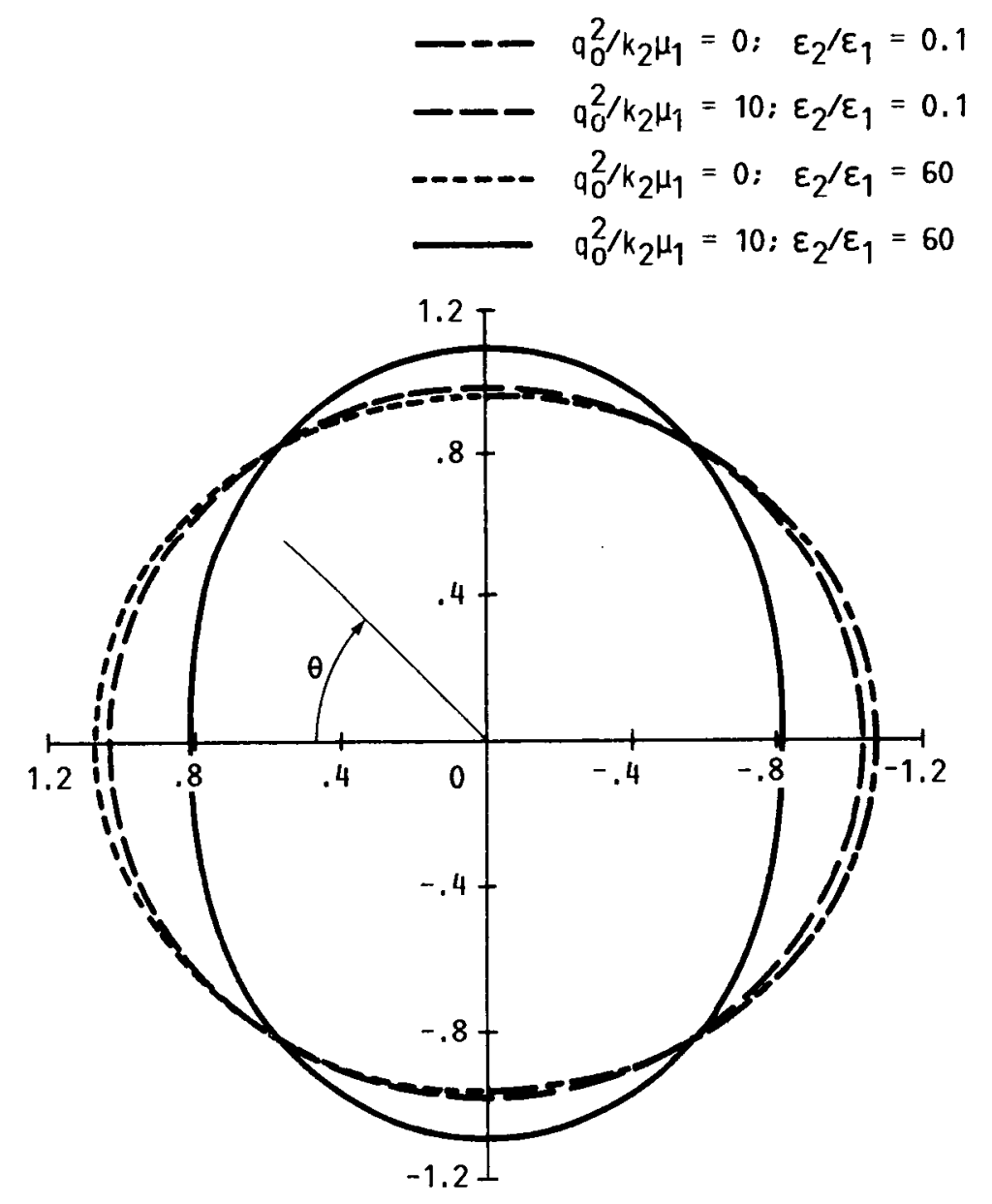

FIGURE 3. - DROPLET SHAPES FOR $\mu_{2} / \mu_{1}=\varepsilon_{1} E_{0}^{2} R_{0} / \sigma_{0}=0.1$. 


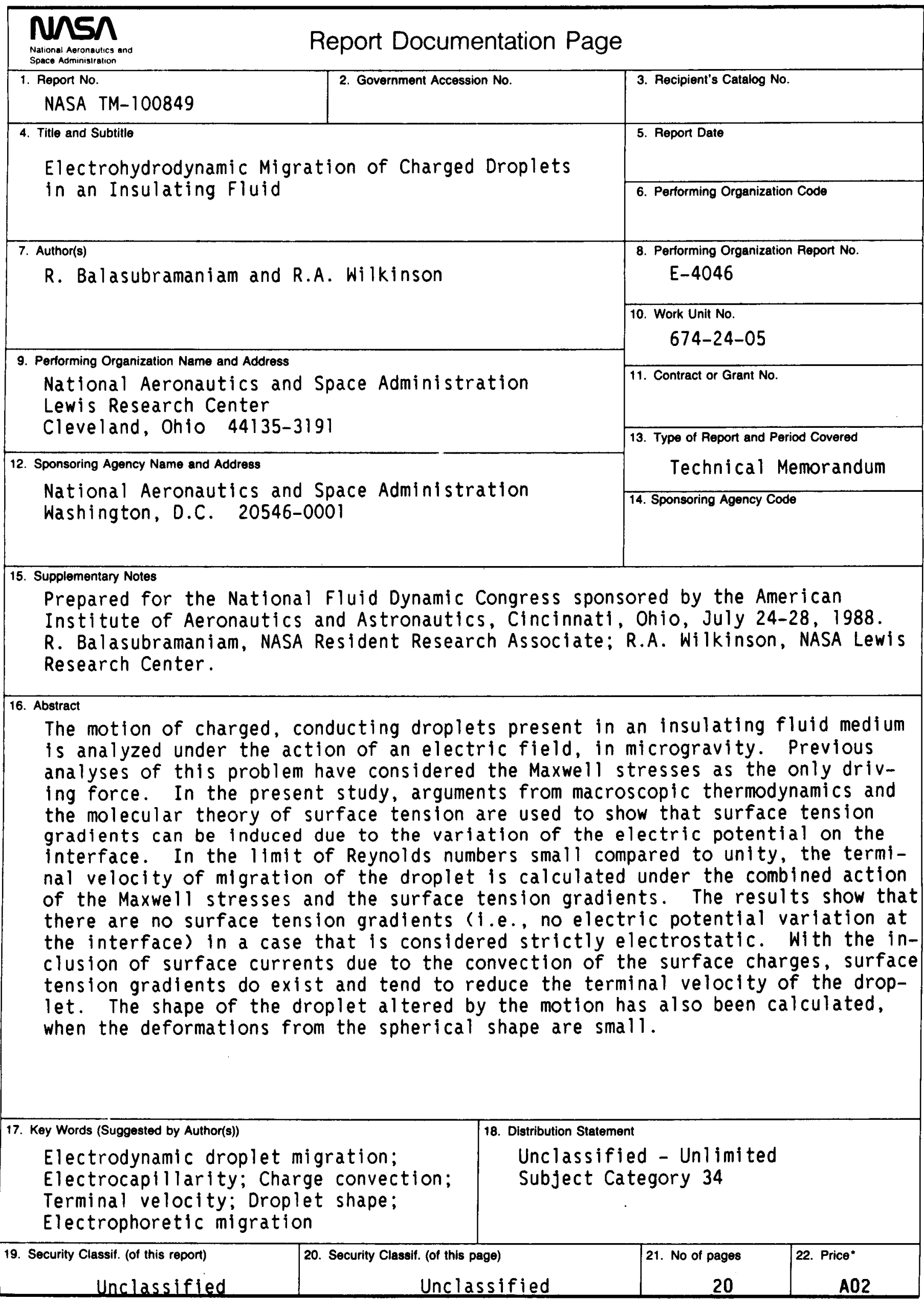

